\title{
Data gathering solutions for dense RFID deployments
}

Abdoul Aziz MBACKE

Inria, aziz.mbacke@inria.fr
Nathalie MITTON

Inria, nathalie.mitton@inria.fr
Herve RIVANO

Univ Lyon, Inria, INSA Lyon, CITI, herve.rivano@inria.fr

\begin{abstract}
The advent of RFID (Radio Frequency Identification) has allowed the development of numerous applications. Indeed, solutions such as tracking of goods in large areas or sensing in smart cities are now made possible. However, such solutions encounter two main issues, first is inherent to the technology itself which is readers collisions, the second one being the gathering of read data up to a base station, potentially in a multihop fashion. While the first one has been a main research subject in the late years, the next one has not been investigated for the sole purpose of RFID, but rather for wireless adhoc networks. This multihop tag information collection must be done in regards of the application requirements but it should also care for the deployment strategy of readers to take advantage of their relative positions, coverage, reading activity and deployment density to avoid interfering between tag reading and data forwarding. To the best of our knowledge, the issue for a joint scheduling between tag reading and forwarding has never been investigated so far in the literature, although important. In this paper, we propose two new distributed, crosslayer solutions meant for the reduction of collisions and better efficiency of the RFID system but also serve as a routing solution towards a base station. Simulations show high levels of throughput while not lowering on the fairness on medium access staying above $85 \%$ in the highest deployment density with up to $\mathbf{5 0 0}$ readers, also providing a $90 \%$ data rate.
\end{abstract}

\section{INTRODUCTION}

The evolution of RFID technology has helped improve various applications that were traditionally limited by the tools in use. As such, in stores and supply chains, the use of passive RFID attached to goods helped improve productivity and tracking. The ability for RFID readers to access the data stored in tags without direct line of sight also increases the ease-of-use and makes the application transparent to the naked eye. Another application that could be improved thanks to RFID is sensing in smart cities. While nowadays sensors are used to remotely collect data (temperature, pressure, pollution, humidity etc) in the environment, the recent advances in RFID could provide better and more energy efficient solutions. In fact, modern sensors necessitate a battery to properly gather measurements and send it to the base station, which in turn limits their lifetime. In order to get proper measurements, these sensors would need to be buried or embedded in concrete or similar materials but this would degrade their communication and impact the quality of the system. Under such constraints in terms of battery replacement of sensors and communication degradation, RFID provides a much better tradeoff. Indeed, deploying passive RFID sensing tags [1] embedded in urban

This work was partially supported by CPER DATA and IPL CityLab@Inria. infrastructures which would be accessed by readers scattered throughout the city or mounted on public transportation makes the system more autonomous in terms of energy but also more resilient to the environment dynamics.

However, the use of RFID comes at not negligible costs due to radio medium in use. In fact, large deployments of RFID readers in close proximity generates reading collisions that affect the system by missing tags due to interference. This issue has been tackled by many and various solutions. Using RFID instead of traditional wireless sensors also induce the need of a communication layer between readers in order to collect data towards a base station in a multi-hop fashion between readers. Unfortunately, no current solution provides a complete working algorithm. Having a joint operation benefits from the deployment strategy of readers to offer more reliable applications and avoid cross interference between both phases (reading and forwarding). The number of tags read by each reader in its vicinity defines how often it needs to share its information, which impacts the energy consumption. Also readers in denser deployment areas could benefit from the redundancy of their tag information to optimize channel use. While an "off-the-shelf" wireless data collection solution applied to an RFID systems would not be able to care for the specification of both the application and the readers parameters. In [2], three distributed TDMA approaches were introduced to alleviate the issue. They were all compared to a state-of-the-art solution and provided much better performance in visited metrics. Based on the characteristics given, we retained two algorithms that will be used as our starting point to propose a complete data gathering solution for RFID.

In this paper, we propose two new complete integrated RFID anticollision-gathering solutions. The first one is based on mDEFAR which is an efficient monochannel RFID anticollision scheme and the second one is based on CORA which is also a monochannel anticollision algorithm but intended to reduce coverage delay. Since both algorithms are TDMAbased, the idea is to observe the activity of readers and based on their chosen slot, have them forward their readings towards the base station. This forwarding mechanism will follow a previously built gradient topology on the deployment, defining a rank for each reader. We thus propose two joint readinggathering mechanisms for a multihop RFID reader network that features the following characteristics :

- distributed and local: each device should run the same algorithm relying solely on the information given by neighbors 
in its vicinity, allowing for the solution to be scalable ;

- efficient: ensuring that each reader gets fair access to tags and improves throughput while decreasing collisions and latency ; - reliable: providing a dependable data rate in terms of multihop data collection with low latency.

The rest of this paper is organized as follows. Section II reviews the problem statement and highlights our motivation, Section III introduces our proposals and details their operating scheme. Performance evaluation on both anticollision and data collection is presented in Section IV and Section V discusses the related work. Section VI raises the perspective of this work and future improvements before concluding in Section VII.

\section{Problem Statement}

\section{A. Dense environments}

Large deployments come at the cost of radio collisions which deteriorate the performance of RFID systems. Indeed, collisions in RFID can result in failure to identify tags. The contention resolution is here different from the traditional Wireless Sensor Networks (WSN) schemes where nodes actually interfere between each other with their communication range overlapping. Here RFID readers are not indeed colliding themselves but interfering over the tags they cover.

\section{B. Dedicated control channel}

In order to accommodate readers and avoid reader collisions, a solution could be to have them read at different times following a TDMA-based algorithm. However, such a solution requires readers to communicate either between themselves or with a superior entity such as a central server meant to organize readings. Whichever solution is retained, a constant precondition is the use of a dedicated communication channel apart from the interrogation channel. This communication channel can either rely on a wired or wireless medium. The hypothesis of a communication/control channel is very present in literature and has been made both for centralized [3] and distributed schemes [4]-[6]. In view of the scenarios given in I, we opted for a distributed scheme using a wireless communication channel. We figured the communication range $d_{C o m}$ for this channel should at least be twice the interrogation channel range $d_{C R T}$, as such $d_{C o m}=2 \times d_{C R T}$. This value ensures that readers that agree on the communication channel are far enough from each other not to collide and decreases the chance of "hidden terminal" issues as well.

\section{Data gathering}

While ensuring that readers activity do not interfere and that tags are successfully identified, the way the information being read is sent in parallel to the base station should also be considered. Indeed, most solutions reviewed only consider the anticollision issue, in [3], authors briefly discuss the architecture of mobile RFID readers transmitting the tags information towards a central server. But this property is not investigated afterwards. In some WSN, nodes are expected to carry a long range communication device allowing them to reach a base station located miles away. However in our proposals we took advantage of the already present dedicated communication channel to relay tag information between readers on top of a gradient topology. We believe that the design of an anticollision scheme must also take account of the collection of tag information to optimize readers activity, predict the energy consumption and prevent data reading and forwarding phases to interfere. Both our proposals modified the initial behavior of their algorithms [7] to integrate the feedback activity.

\section{PROPOSED ALGORITHMS}

In this paper, we aim at introducing improved solutions to both reduce reader collisions as well as to relay tag information to a base station. In order to get great performance of the system, we designed fair and rapid coverage solutions.

\section{A. Background}

The full algorithm of mDEFAR and CORA can be found in [7], the modifications made to include data relaying will be explained in the next sub-section. However, the main difference between mDEFAR and CORA is how they handle collisions. While mDEFAR mainly focuses on decreasing the collisions, CORA maximizes the throughput by having multiple readers access tags at the same time. Indeed in mDEFAR, only a single reader is selected in its vicinity based on its ID and priority level. This ensures collision-free operations. In CORA, readers observe their vicinity and depending on the number of potential colliding neighbors decide either to read or skip the current operation. As such, multiple readers can simultaneously access tags, colliding over a given number of tags that will be covered during following operations.

\section{B. Gradient construction}

In order to ease the relay of tag information between readers and toward a central sink, we rely on a gradient topology. This gradient topology assigns a rank to each reader, which is later used to determine the reading slot in the collection process. Algo. 1 describes the method followed by readers and the sink. The sink broadcasts a beacon with Rank 0 using the dedicated control channel. Upon reception of a beacon, a reader checks if the rank contained in the beacon $\operatorname{rank}_{R X}$ is lower than its own rank rank $k_{\text {local }}$. If so, the reader considers the source of the beacon as its new "parent" ( parent $\left._{\text {local }}\right)$, sets its rank as rank $_{\text {local }}=\operatorname{rank}_{R X}+1$ and draws a random backoff before broadcasting a beacon at its turn with its

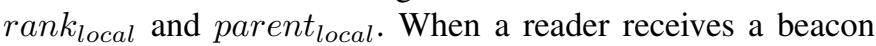
with a parent $_{R X}$ corresponding to its Id, it considers the source of this beacon as one of its "children". This random backoff allows readers not to collide while sending beacons. Eventually, all readers have a rank designated by their hop distance to the sink and a preferred "parent".

Fig. 1a, shows an example of a topology. Links between nodes show communication links. At $t=0$, the base station sends a beacon with rank 0 (Algo 1, lines 1- 3). At reception, readers $\mathrm{A}$ and $\mathrm{B}$, which are in range of the base station, resp. set their ranks to 1 and save the base station as their direct parent. At $t=1$, reader $\mathrm{A}$, that drew a lower backoff than 


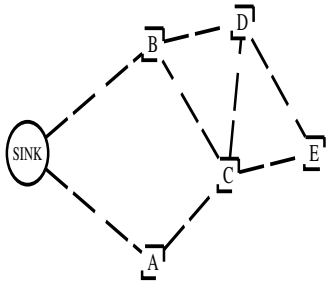

(a) $\mathrm{t}=0$

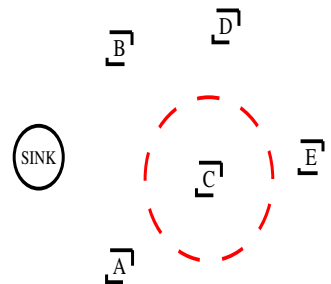

(b) $t=0$

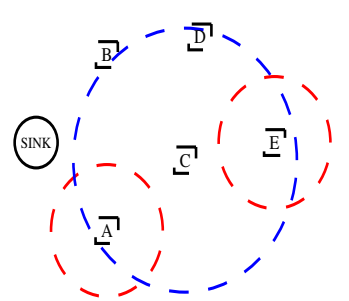

(c) $t=1$

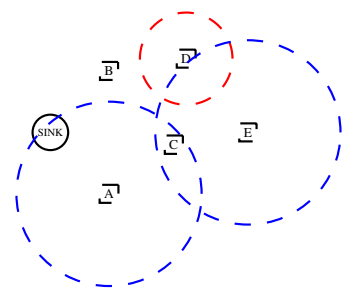

(d) $t=2$

Fig. 1: Gradient construction, reading and data collection

$\mathrm{B}$, sends its beacon which is received by reader C. C sets its rank to 2 and saves $\mathrm{A}$ as a parent before forwarding too. At $t=2$, C forwards its beacon which is received by A that save $C$ to its children list(Algo 1, lines 12 - 14), and also by $\mathrm{B}, \mathrm{D}$ and $\mathrm{E}$. $\mathrm{B}$ which already has a lower rank than the one received discards the beacon (Algo 1, line 8), D and $\mathrm{E}$ receive the beacon and set their rank to 3 . At $t=3$, B finishes its backoff and forwards the beacon with rank 1 . It is received by $\mathrm{C}$ and $\mathrm{D}$. C which already has a rank 2 discards the beacon (Algo 1, line 8), but $\mathrm{D}$ that previously had a rank 3 , now sets its rank to 2 and sets B as its parent. When D then forwards its beacon, it is received both by B, C and E. B saves D as child, $\mathrm{C}$ and $\mathrm{E}$ both discard the beacon since $\mathrm{C}$ already has a rank 2 and $\mathrm{E}$ also has a rank 3 (Algo 1, line 8).

\section{Data gathering}

The collection of tag information and relay towards the sink is done in synchronization with tag interrogation. In order to spare readers activity and reduce collisions, we set $\max _{\text {slot }}=$ $\max _{\text {rank }}$. In both, mDEFAR and CORA, instead of having readers randomly chose their slots between $\left[0 ; \max \_s l o t s[\right.$, they now chose a slot based on their rank (see Algo. 2). As such, readers with an even rank $\left(\operatorname{rank}_{\text {local }} \% 2==0\right)$, will randomly chose an even rank between $[0 ;$ max_slots [, readers with an odd rank ( rank $\left._{l o c a l} \% 2 !=0\right)$, will randomly chose an odd rank between $[0 ;$ max_slots[. Two readers on two consecutive ranks will not be able to read on the same slot, decreasing the contention. And also two readers on the same rank may not chose the same slot since they chose randomly.

After choosing their slots, readers perform accordingly to the respective anticollision algorithm (mDEFAR or CORA), with odd readers reading in odd slots and even readers reading in even slots. This gap between even and odd readers is then used to relay data. Indeed, after interrogating tags during an even slot, a reader then forwards tag information to its parent on the following (odd) slot. Since the dedicated control channel is different from the tag interrogation channel, these transmissions will not interfere with the readers normal activity. Independently from the issue of the contention, three outcomes are possible (see Algo. 3):

- slot $_{\text {local }}<m a x \_r a n k-1$ AND pkt_to_f $f d_{\text {local }}>0$, the reader has tag information to transmit either obtained from its reading activity or from his children activities. The reader wakes up at slot $n+1$ and transmit tag information to its parent during $T_{C R T}$ and then waits for the following frame.
If the tag information has not been fully transmitted, it will be continued on the next relay occasion ;

- pkt_to_f $w d_{l o c a l}==0$, the reader has no tag information to forward to its parent. It waits for the next frame to perform its contention according to the chosen algorithm ;

- slot $_{\text {local }}==m_{a x} \_r a n k-1$ AND pkt_to_f $w d_{\text {local }}>0$, the reader performed its tag interrogation in the last available slot of the current frame and has tag information to forward. It waits for the following frame, performs the contention algorithm chosen and according to the new slot $_{\text {local }}$ chosen, will forward its data.

Figure 1 shows the continuation of the previous example. After setting their ranks, nodes can now begin their reading and data collection activity. At $t=0$, reader $\mathrm{C}$ which has an even rank value can chose Slot 0 (Algo. 2 lines 1 - 5). $\mathrm{C}$ then proceeds to read tags within its interrogation range $d_{C R T}$ defined by the red dotted circle. After tag interrogation, it waits for the next slot. At $t=1$, A (rank 1) and $\mathrm{E}$ (rank 3), can then proceed to read if they chose the same slot (Algo. 2 lines 6 -9). From this observation, we can see that the choice of having odd ranked readers reading at odd slots and same for the even ranked readers allows the reduction of potential collisions. As such, A and E can read at the same time without any risk of collision. At the same time, $\mathrm{C}$, that read previously, can now forward its data (blue dotted line) to its parent A (Algo. 3 lines 1 - 8). Since this communication is happening on a dedicated control channel, different from the interrogation channel, it does not interfere with the interrogation of A or E. At $t=2$, even ranked reader $\mathrm{D}$ can now access tags. While it accesses tags, both $\mathrm{A}$ and $\mathrm{E}$, can forward their data to their respective parents. Reader A can forward both the tag information it read but also the tag information forwarded by $\mathrm{C}$ (Algo. 3 lines 14 - 17) towards the sink. E also can forward its data to its parent C. Communications of A and $\mathrm{E}$ do not collide on reader $\mathrm{C}$ since a backoff is randomly drawn before forwarding. Since max_rank $=3$ according to the given example, the current frame ends after Slot 2 (Algo 2). As such, reader D that previously accessed tags on Slot 2, now has to wait for the next frame before forwarding its tag information (Algo. 3 lines 11 - 13). Reader B that did not read, nor receive data from its children, has no tag information to forward, it then waits for the next frame (Algo. 3 lines 9 - 10). 


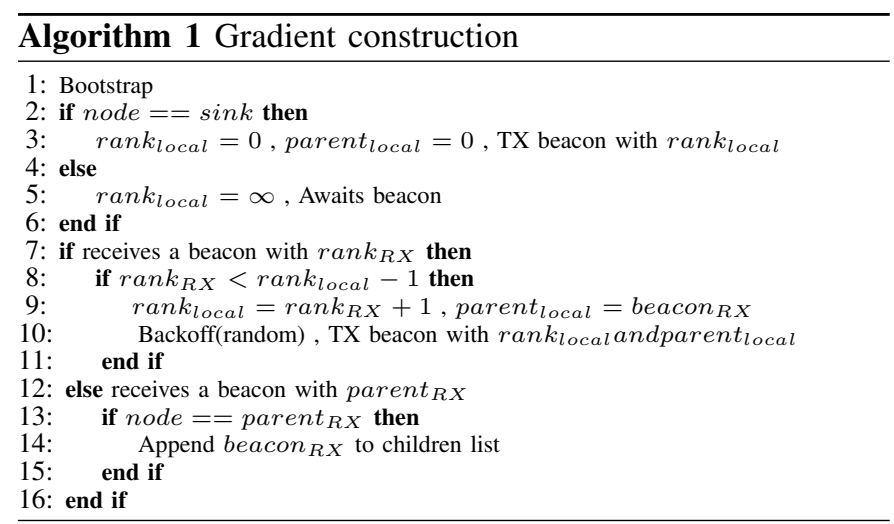

\section{PERformance Evaluation}

In order to evaluate the performance of our proposals, they were simulated using WSNet. We ran simulations with from 100 to 500 readers, to validate the algorithm under different constraints on density. The values of $T_{\text {Beacon }}$ and $T_{C R T}$ were respectively set to $5 \mathrm{~ms}$ and $460 \mathrm{~ms}$ according to [8]-[11].

\section{A. Anticollision}

1) Throughput: or average number of Successful Query Sections (SQS) i.e. number of successful contentions won by the reader which can access the medium. The higher the throughput, the faster the tag information is retrieved. Fig. 2a shows the throughput results of our solutions. We can see that CORA, thanks to it contention resolution mechanism, offers higher values. While mDEFAR tends to keep only one reader enabled, multiple readers can be enabled in CORA as long as there are other devices on different parameters.

2) Collisions: In the case of mDEFAR, collisions are counted when a reader fails to send its beacon due to backoff collision or when a reader is disabled after the contention process. While in CORA, collisions are counted in backoff course but purposely collisions defined by the algorithm are accounted for as well. In Figure 2b, we can see that, while CORA offered a better throughput, the tradeoff in terms of collisions is obvious. As such, as the number of reader grows in the system, the number of collisions increases accordingly. However while a collision in mDEFAR means involved readers are disabled, in CORA a collision just means that tags laying in overlapping interrogation range will not be identified.

3) Jain's Fairness Index (JFI): Since readers are randomly deployed, it is relevant to know how fairly each reader gets access to the medium. Indeed the fairer the protocol, the better

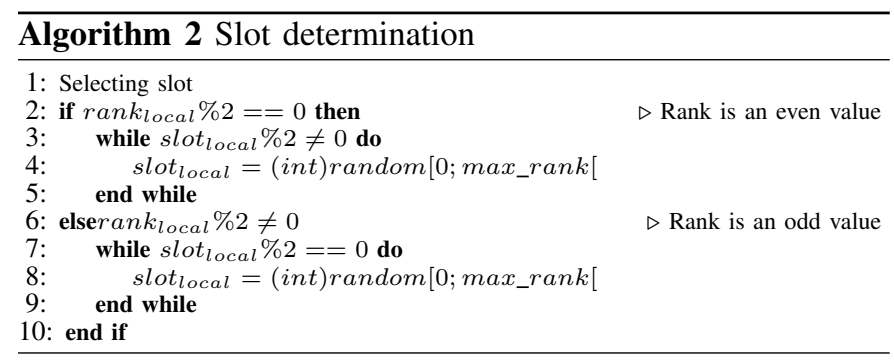

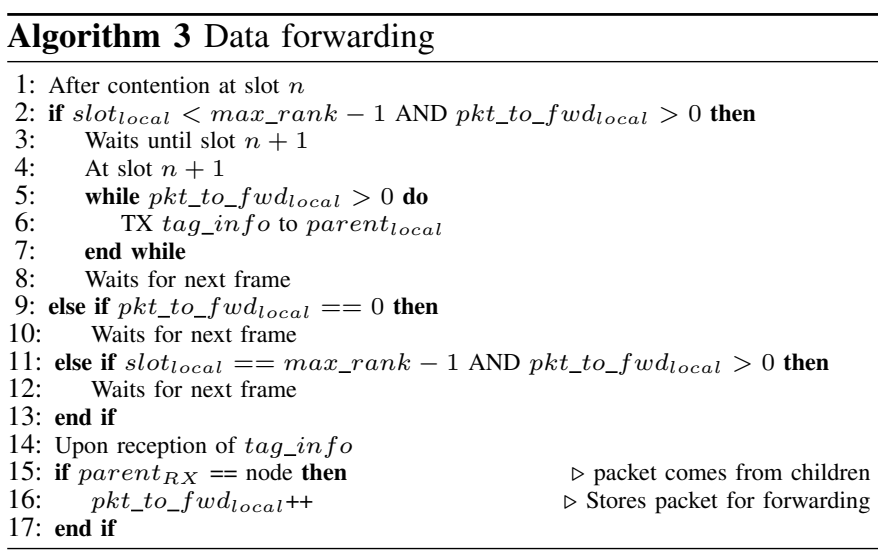

the medium is shared and tags are fairly being identified. Figure 2c shows the fairness results of our proposals. While CORA, thanks to its contention resolution process, offers better fairness than CORA, they both are quite performing since the fairness always is over $85 \%$. This means that whichever solution is chosen, the access to the medium will remain fair and tags will be evenly read.

4) Coverage delay: This metric gives the time needed to cover all tags within the deployment area. In Figure 2d, only the results with 500 readers are considered. We can see that after the first round, already more than $75 \%$ of tags are already covered using either algorithm. and that after 20 rounds, all the tags have already been read at least once. This proves that all tags within deployment range are fairly being serviced. One other interesting fact to notice is that the tradeoff made by CORA regarding collisions to offer better latency is indeed valid since the coverage is slightly quicker.

\section{B. Data collection}

In order to assess the performance of data collection, we considered two metrics:

1) Packet data ratio (PDR): This metric shows the percentage of message that are successfully transmitted to the base station. It is computed as the ratio of the number of tag information received at the sink over the total number of tag information read and sent. In Figure $3 a$, the results of our simulations are displayed. While the PDR stays consistently over $85 \%$, mDEFAR offers better results never falling under $92 \%$. This can be explained by the fact that mDEFAR with its lower throughput in turn has a more stable system to perform better in the data collection forwarding. While the high number of SQS in CORA generates a large number of tag information data to be sent and induces this lower PDR.

2) Latency: The latency assesses the delay between a tag information being read and its reception by the base station. in the case of our algorithm, since readers are organized in a gradient topology, the latency is given according to the rank of the source reader. As such on Figure 3b, the average latency, in seconds, for each considered rank with a 100 readers deployed is shown. We can see that even at the $6^{\text {th }}$ rank, a tag information takes about 13 seconds to reach the 


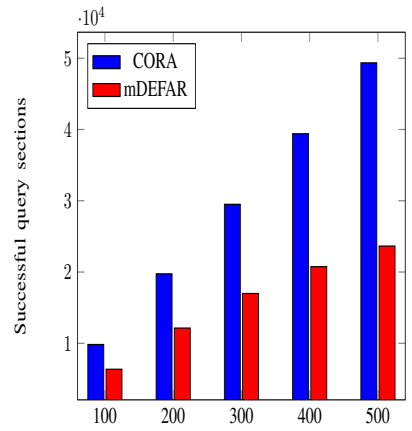

(a) Throughput

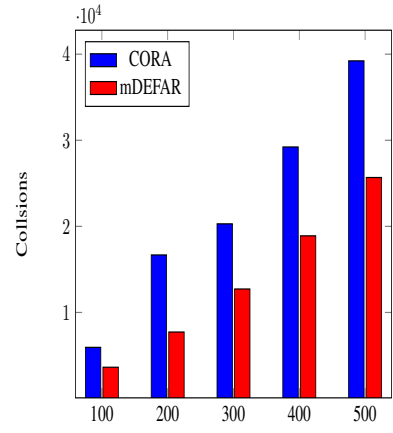

(b) Collisions

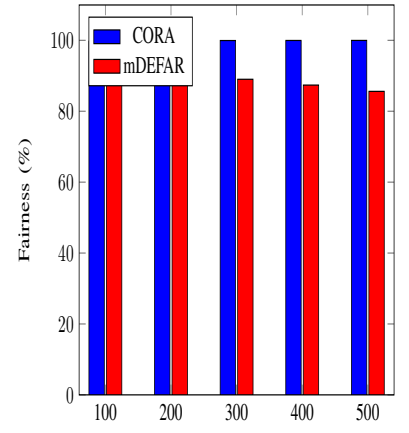

(c) Fairness

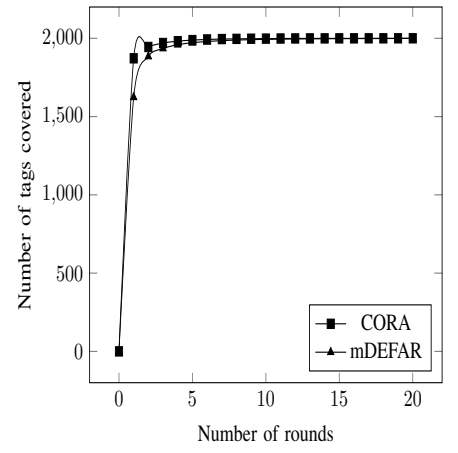

(d) Coverage delay

Fig. 2: Anticollision Results

sink. In the case of a smart city, it means that in case of an alert regarding the state of an urban infrastructure, it would take 13 seconds for authorities to be alerted and react. On Figure 3d, the average latency for 500 readers is shown. We can see that the growing number of readers in turn slightly increased the latency by up to 3 seconds (around 16s). This is due to the high density of readers and their high activity producing more congestion. As discussed in VI-A, we believe that introducing new metrics other than the hop count could help improve the performance by granting new routing options.

\section{RELATED WORK}

Extensive work has been undertaken to improve RFID systems through collision resolution. Most of these works can be classified either as TDMA or CSMA-based, respectively relying on the synchronization of readers or on the listening of the medium. The standard [8] proposes Listen Before Talk (LBT). As its name suggests, readers willing to interrogate tags ensure that no other reader is active in their vicinity. This "listening" is performed for a defined period of time after which the reader performs a backoff before attempting to check the status of the medium again and then read task if idle, "talk". In [10], authors propose to check the status of the interrogation channel before transmitting and triggering a random backoff in case of occupancy. As a distributed algorithm it allowed to reduce collisions while not relying on a dedicated control channel. Another solution [12] based on differential backoff proposes to have readers exchange beacons after a backoff based on their residual energy level. According to the signal strength at reception, readers then decide whether interrogate tags. A broadcast of tag information is then done by readers, but just for the sake of ensuring failed contenders that the tags in their range were already identified.

Centralized solution can also be found as in [3], which is an improved version of GDRA [9]. After receiving orders from the server to access tags, readers exchange beacons to reserve the slot in their interrogation range. While in GDRA, all neighboring readers would systematically get disabled, their algorithm allow readers to modulate a fairness parameter.

Many additional solutions can be found throughout the literature and could hopefully be adapted to our tag information gathering solution in order to have fully autonomous systems.

\section{IMPROVEMENTS AND FUTURE WORK}

During our work, several questions were raised that we look forward to address in our future works that we discuss here.

\section{A. Metrics}

While in this paper the only metric considered by a reader to forward its tag information is the number of hops towards the sink, we believe that several other metrics should be taken into account to offer a solution that could be validated in real experiments. Indeed, the hop count as considered by our solution cannot be maintained due to the nature of radio medium. As such, we believe that link quality between readers, latency, packet drop rate, energy levels between neighbors should also be considered to propose a more robust solution.

\section{B. Aggregation}

In our simulations, due to the high reader density, we had multiple readers simultaneously covering the same deployed tags. This induces multiple instances of the same tag information being transmitted to the sink. We believe that aggregating/filtering data before forwarding could help improve both the PDR by having less packets being sent but also the latency by lowering the congestion. This compression could be done at the reader's level, using the "parent readers" to match and compress data received from their "children". But since readers receive information from higher ranks at different times, investigation towards the optimal time to perform this aggregation needs to be investigated. For instance, [13] investigates the energy efficiency of distributed compressed sensing in WSNs. Comparing the sensing activity and radio activity, authors then prove that in several applications, compressed sensing offers a better ratio in term of energy efficiency. In [14], authors use the spatial correlation of gathered data in WSNs to propose a reliable compressed sensing solution. However these solutions need to be tweaked to support RFID compressed sensing, since the computation happens, not at tag level but at the reader's, which already aggregates data from all tags in range. Works proposed in [15] suggest RFID tag sensing in stores by leveraging on tag collisions. Although these works are not completely in phase with ours, we believe that they could serve as a starting point to propose a performing aggregation solution. 


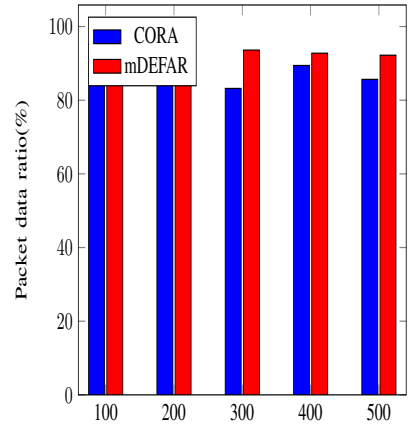

(a) PDR

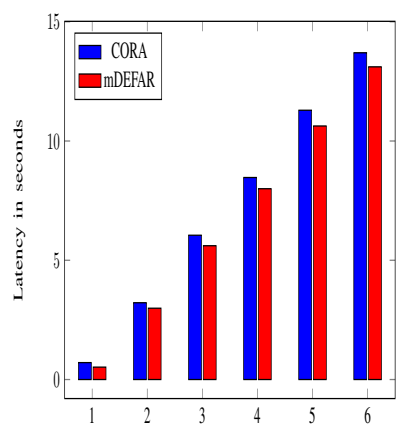

(b) Latency - 100 readers

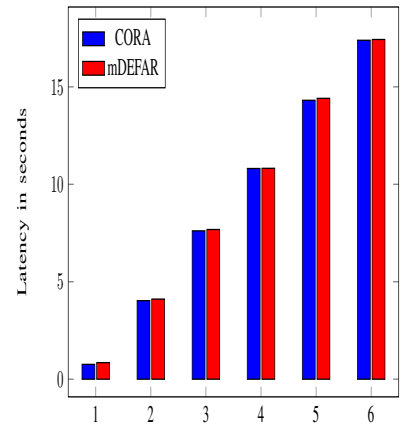

(c) Latency - 300 readers

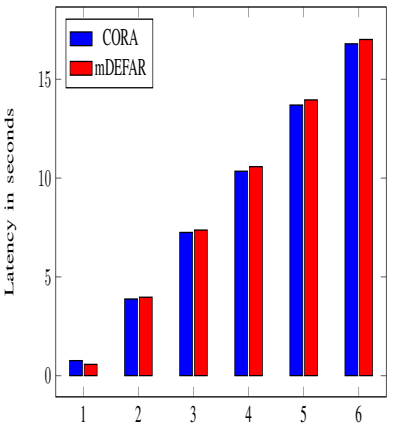

(d) Latency - 500 readers

Fig. 3: Anticollision Results

\section{Mobility}

As proposed in Section I, readers are now used in various scenarios. In those latter, the option of mounting them on mobile entities such as vehicles or giving them to roaming workers on foot is now considered. This could allow for an even easier tracking and better coverage. While the proposed solutions, mDEFAR and CORA, have already been extensively tested as anticollision algorithm in [2] and performed well, compared to the state-of-the-art, they still need to be validated with the data gathering proposed in this paper. One of the main challenges of applying this solution to a mobile scenario is the maintenance of the gradient architecture. Indeed, in a static deployment, where the topology only suffers minor updates, in a mobile environment the topology is constantly changing and the ranking of readers needs to be updated accordingly. In order to maintain the topology the frequency at which beacons are exchanged needs to be modulated in tune with the range of readers, their speed, the environment, etc, as studied in [16].

\section{Energy consumption}

The proposals made in this paper rely on a relatively important exchange of beacons to build the topology and solve reading contentions between devices. Also in an algorithm like CORA that willingly grant collisions for better coverage, the impact of collisions should be measured. In future works, we would like to investigate the energy cost of our solutions and offer better tuning to improve the quality of RFID systems.

\section{E. Data packets}

For the sake of simulations proposed in this paper, we considered generic data packets sizes based on the number of tags covered by readers. We assumed that each tag sent to the reader in range a value of 96 bits corresponding to its EPC [8]. After running these experiments on a RFID-testbed, we plan on offering more realistic data packet sizes based on our observations. Indeed, having non-homogeneous data packet size will impact the data collection, which will be prone to more losses. We intend to evaluate these losses and potentially overcome them. the use of a solution, as discussed in VI-B, will confirm the results of our proposals.

\section{CONCLUSION}

This paper presents two new distributed algorithms meant for data collection of RFID tag information in a multihop wireless RFID network. While they both display promising results, the choice of one algorithm or the other should be guided by the application needs. Indeed, while a better throughput is obtained using CORA, it impacts both the number of collisions as well as the PDR where mDEFAR performs better. Tradeoffs, based on the application, the deployment scenario and the desired performance should indicate the best choice. Next step will investigate topics of Section VI. Experiments on a RFID testbed are considered to confirm simulation results.

\section{REFERENCES}

[1] P. I. Engineering”, "About wireless rfid sensors (battery-free)."

[2] A. A. Mbacke, N. Mitton, and H. Rivano, "Rfid reader anticollision protocols for dense and mobile deployments," MDPI Electronics Special Issue "RFID Systems and Applications", 2016.

[3] H. Rezaie and M. Golsorkhtabaramiri, "A fair reader collision avoidance protocol for rfid dense reader environments," Wireless Networks, 2017.

[4] J. Waldrop, D. W. Engels, and S. E. Sarma, "Colorwave: an anticollision algorithm for the reader collision problem," in Proc. of ICC, 2003.

[5] S. Birari and S. Iyer, "Pulse : A mac protocol for rfid networks," in Proc. of Int. workshop on RFID and Ubiquitous Sensor Networks (USN), 2005.

[6] A. A. Mbacke, N. Mitton, and H. Rivano, "Distributed efficient \& fair anticollision for rfid protocol," in Proc. of WiMob, 2016.

[7] A. A. Mbacke, N. Mitton, and H. Rivano, "Rfid anticollision in dense mobile environments," in Proc. of IEEE WCNC, 2017.

[8] EPC TM radio-frequency identity protocols class-1 generation-2 UHF RFID protocol for communications at $860 \mathrm{MHz}-960 \mathrm{MHz}$ version 1.2.0, EPCglobal Standard specification Std. 1.2.0, 2007.

[9] V. Bueno-Delgado, R. Ferrero, F. Gandino, P. Pavon-Marino, and M. Rebaudengo, "A geometric distribution reader anti-collision protocol for RFID dense reader environments," IEEE T-ASE, 2013.

[10] I. Amadou and N. Mitton, "HAMAC: High adaptive MAC protocol for dense RFID reader-to-reader networks," in Proc. of AdHocNets, 2015.

[11] I. Amadou, A. A. Mbacke, and N. Mitton, "How to improve csma-based MAC protocol for dense RFID reader-to-reader networks?" in Proc. of ADHOC-NOW, 2014.

[12] M. Golsorkhtabaramiri, M. Hosseinzadeh, M. Reshadi, and A. Rahmani, "A reader anti-collision protocol for rfid-enhanced wireless sensor networks," Wireless Personal Communications, 2015.

[13] M. A. Razzaque and S. Dobson, "Energy-efficient sensing in wireless sensor networks using compressed sensing," MDPI Sensors, 2014.

[14] C. Luo, F. Wu, J. Sun, and C. W. Chen, "Compressive data gathering for large-scale wireless sensor networks," in Proc. of MOBICOM, 2009.

[15] M. Mayer, N. Görtz, and J. Kaitovic, "Rfid tag acquisition via compressed sensing," in Proc. of RFID-TA, 2014.

[16] F. Ingelrest, N. Mitton, and D. Simplot-Ryl, "A turnover based adaptive hello protocol for mobile ad hoc and sensor networks," in In Proc. of IEEE MASCOTS, 2007. 